\title{
COLLABORATION ENHANCES COMMUNITY WELLBEING: A COMMUNITY DEVELOPMENT RESEARCH FRAMEWORK
}

\author{
Jean Ross, Samuel Mann and Keith Whiddon
}

\section{INTRODUCTION}

This paper describes a collaborative application of the Community Health Assessment Sustainability Education (CHASE) model (Ross, Crawley \& Mahoney, 2017) to rural community development. The rural population nationally and internationally accounts for almost half of the total global population and these communities are confronted with reduced access to healthcare which contributes to increased health disparities (Couper et al., 2015; Hogarth \& Rapata-Hanning, 2015). Nurses play a critical role in reducing these disparities around the globe with limited models to guide their practice (Ross \& Crawley, 2018; Doolan-Noble, 2019). While nurses are positioned to navigate the holistic landscapes of health that integrate the socio-political, cultural, sustainable, economic and environmental aspects related to the uniqueness of rural communities they, require models to guide their practice. The CHASE model is one such model that guides practice however, we are aware one such model does not fit all communities therefore, the purpose of this research is to revise how this model is used and adapted for ongoing and future application.

The purpose of this paper is to demonstrate the value of collaboration and contribute to the ongoing debate about collaboration, health, community partnership and sustainability. This is part of a larger research project and we use the framework of this to structure this paper (Figure I). The collaborative approach is illustrated with the example of a case study connected to the rural community of Bishop's Castle, Shropshire, England, UK. This community project was conducted in February 2020 (pre COVID-19) in collaboration with student nurse learners from New Zealand (NZ), supervised by the first author in partnership with the third author, who is a resident of this community and is the Chair of the Bishop's Castle Community Partnership. The core focus of this project was to identify and improve the health needs of the rural population of Bishop's Castle in collaboration with local community development practitioners (all members of the community), educators and researchers. We bring together these elements associated with the practice of community research by providing a discussion and practical guidance on the use of the CHASE model in learning and teaching.

\section{COLLABORATIVE COMMUNITY RESEARCH FRAMEWORK}

The Bishop's Castle collaboration is aligned with the global community development research project titled Globalising the teaching and learning CHASE model to enhance nursing education: A national and international comparative systematic participatory data analysis, we are currently progressing. This global research project is based on a research framework of six separate but interlinking elements, presented diagrammatically in this paper in Figure I which highlights how these elements (emphasised by six shades of blue) are comprised in this research project, stressing the collaborative relationships between and with community, education, research, co-design, evaluation and the ongoing and future collaborations within the local to global contexts. The image of the globe in Figure I provides a central focus to this framework, representing collaborative relationships expressed by the numerous hands, placed around the global connecting with the six distinct elements of the Community Development Research Framework. These elements are introduced and exemplified as a component of this 
case study. We bring together these elements associated with the practice of community research by providing a discussion and practical guidance on the use of the Community Health Assessment Sustainability Education (CHASE) (Ross, Crawley \& Mahoney, 2017) model in learning and teaching (led by the second author). This research is a unique partnership between local NZ providers aligned with education, health and research; international regions and organisations, and international participating Schools of Nursing and communities. Ongoing collaborative relationships are being developed and for 2021 this project has extended an invitation and will be working in partnership with Global Engagement and the College of Creative Practice and Enterprise both situated at Otago Polytechnic, Dunedin, NZ and are engaged with the Design for Social Innovation and Sustainability Lab (DESIS Lab) an international co-design collaborative practice enterprise illustrated in Figure I and further discussed in the following sections representative within this framework.

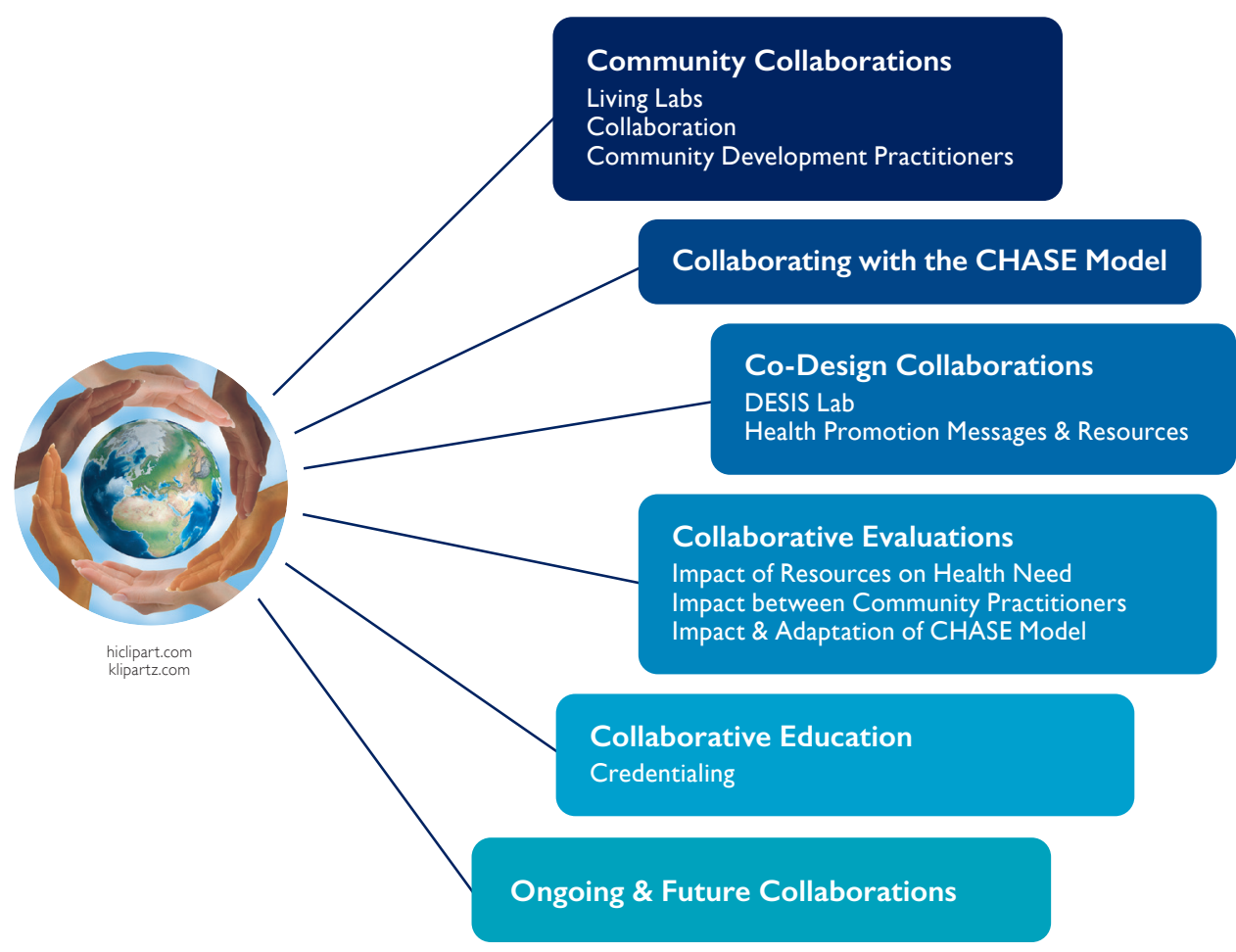

Figure 1: Community Development Research Framework. Source: Authors

\section{CASE STUDY}

Nurses act as community development practitioners (International Association for Community Development, 20 I8) to improve health outcomes in partnership with community stakeholders. It is therefore imperative student nurse learners experience community development work, as part of their primary health care clinical placements. Through the School of Nursing, Otago Polytechnic, Dunedin, NZ these learners have engaged with an education and research team, who profiled the rural English town of Bishop's Castle, Shropshire, UK and worked in collaboration with members of this community, with the aim to identify and reduce health disparities. Further elaboration of this case study including the student's community development research is published in this Journal (Restieaux et al., 2020) providing an in-depth review of the health needs identified and the outcomes of the research project. 


\section{Community Collaborations - the first section of the framework \\ Living Labs}

Living labs are spaces where educational, research and health care institutions partner or in other words collaborate at the macro level to enable empowerment of the identified community otherwise considered the case study (BergvallKaeborn, et al., n. d.). This is the level at which the local, national and global political dynamics related to policy, power and influence, are acknowledged and taken into consideration. While at the micro level activities are undertaken to highlight the community assets, deficits and capabilities, with a view to harnessing innovation and empowerment.

\section{Collaboration}

Collaboration involves groups of individuals working together on a project. All individuals involved in this project are partners and they have shared in the workload. Sharing the work includes clear insights, purpose, ideas and process to achieve a common objective and create new understandings and visions (Gilbert, 20l6). Collaboration between all the engaged participants are referred to in this paper as community development practitioners.

\section{Community Development Practitioners}

Community development practitioner is an empowering phrase to classify all members of the community project whether in a paid or un-paid capacity (International Association for Community Development, 20 18). Core community development values guide and underpin their practice including commitment and dedication to partner together with respect of different ideas and cultures; being patient and mindful of responsive communication while maintaining ethical practice with the aim to improve health status through change, empowerment and education. For the purposes of this case study an ethical application and approval had been sought and accepted from the School of Nursing Ethics Committee, Otago Polytechnic, Dunedin, NZ.

\section{Collaborating with the CHASE model - the second element of the framework}

The CHASE model is the research method engaged with to generate primary and secondary data that applies community development values with the aim to empower communities and their local organisations to sustain and improve health care and comprises six phases. This model comprises six phases. Engaging with the CHASE model (Ross, et al., 2017) provides community development practitioners, in this case study the student nurse learners, a method to profile the community, apply a needs assessment, identify health needs and develop resources (as a codesign collaborative team) with the purpose to improve sustainable health care and reduce health disparities. This model guides community development practitioners to view health from a sustainable and population-based approach to build effective and empowering relationships and consider the unique aspects and attributes of a community.

\section{Co-Design Collaborations - the third element of the framework DESIS Lab}

DESIS Lab is the platform for the Design for Social Innovation and Sustainability Lab, relating to an action research laboratory. DESIS Lab is a collaborative effort connecting 'strategic and service design, management, and social theory, applying interdisciplinary expertise in problem setting and problem solving to sustainable practices and social innovation' (Parsons DESIS Lab, n.d., n. p.). Sustainability is the focal point in which innovative design-led practices work collaboratively within the local and global contexts (Parsons DESIS Lab, n.d.).

\section{Health Promotion Messages and Resources}

Health promotion messages and resources were developed which harnessed and included local members of the core community team who engaged and collaborated with the student nurse learners through the ongoing provision of these resources. They have built up an ongoing and lasting local response to new developments in the community through availability of producing resources. For example, local vegetable boxes including the resources created by the learners are now included with the food distributed by the local food bank and include a fridge magnet and coaster, delivering consistent education on the benefits of a community garden, to improve health and healthy living. Also continuing with community meetings and maintaining ongoing relationships with the nurse learners and the education institution, demonstrating ongoing collaborative relationships. 


\section{Collaborative Evaluations - the fourth element of the framework}

After completion of the community development research project phases one-five of the CHASE model an impact research evaluation (phase six of the CHASE model) begins the process of investigating the outcomes of the project:

\section{Impact of the resources on the identified health needs}

The identified community development practitioners and recipients of the health promotion messages and resources were invited to evaluate if there had been any noticeable improvement of the identified population's health need. The community response to the project findings was overwhelmingly positive. However, COVID-19 lockdown occurred before the resources developed by the learners could be implemented, so much is work in progress. Saying that, the pandemic has brought new opportunities and the lessons learned though participating in the CHASE model will help to shape the community's post COVID-19 recovery plan. Identifying the community's health needs through this project is the first step in lobbying for improved support. These evaluations provide an opportunity to gather themes related to the identified health needs, resources developed and the utilisation these have had on the reduction of health disparities and the improvement of one or more of the United Nations 17 Sustainable Development Goals. (Sustainable Development Goals, n.d.).

\section{Impact between the community development practitioners}

All the community development practitioners expressed the project as being an incredible experience. The members of the Bishop's Castle community were warm and inviting, extremely helpful and welcoming to the student nurse learners. The student nurse learners learnt so much about a different culture, while having the privilege to partner with the community via a virtual platform involving zoom, email and photo image to collaborate with the local community members and stakeholders (a new approach in terms of research for nurse learners, it has been an important learning and insight into community health development in which student nurse learners grew in their knowledge).

\section{Impact and adaptation of the CHASE model}

The CHASE model is recognised internationally as a valid tool and worthy of adapting to meet local and global individual community needs. This model has enhanced student nurse learners' collaborative relationships in the global landscape and further contributed to inform the nursing curricula, teaching and learning pedagogy nationally and internationally which encourages global diversity and invites multiple perspectives to improve decision making and progress on issues that matter to rural nurses and rural communities around the globe.

\section{Collaborative Education - the fifth element of the framework}

Further engagement with Otago Polytechnic Credentialing in relation to Practice in Sustainable Development Goals: endorsed in Good Health and Wellbeing, Zero Hunger, Quality Education; Sustainable Cities and Communities, No Poverty and Partnerships of the Goals - as an example. By completing phase one-five of the CHASE model (the community research project), each student will be granted their capabilities micro-credentials (known as EduBits) and SDG EduBits - as a package/certificate. Micro-credentials acknowledge the achievement of a defined set of skills and knowledge (New Zealand Qualifications Authority, n.d.).

Ongoing \& Future Collaborations - the sixth element of the framework

An International Community Development Symposium hosted in 2022 by Otago Polytechnic will bring together all of the international fellows associated with this research project to engage in a virtual symposium and establish a Global Centre for Community Sustainable Resilience which could work collaboratively with the DESIS Lab. In addition, we are compiling a book/virtual exhibition to capture our international community work related to the adapted CHASE model, data analysis, and impact assessment evaluations and engagement with the United Nations 17 Sustainable Development Goals (Sustainable Development Goals, n.d.).

\section{CONCLUDING COMMENTS}

This paper has described the importance of the application of engaging with and adapting the CHASE model (Ross, et al., 2017) for rural community development, captured in a research process. This process illustrated in Figure I the Community Development Research Framework draws attention to a holistic landscape for community health, with the example of a case study connected to the rural community of Bishop's Castle, Shropshire, England, UK. The research framework integrates six elements comprised in this research project, stressing the collaborative 
relationships (the first element of this research framework) between and with community, education, research, co-design, evaluation and the ongoing and future collaborations within the local to global contexts.

The CHASE model is one such model that guides nurses' practice (the second element of this research framework) while raising student nurse learners' awareness of community development in healthcare contexts and demonstrating this work can impact positively, on health outcomes and reduce disparity (the third and fourth elements of this research framework). Equally the nurse learners engage with the UN 17 Sustainable Development Goals to aid community resilience as collegial international multidisciplinary engagements, as their findings are disseminated (the fifth element of this research framework). Correspondingly, it is the purpose of this research to revise how the CHASE model is used and adapted for ongoing and future application and engagement in local to global contexts (the fourth and sixth elements of this research framework).

Jean Ross (D0000-0003-2467-9233) RN, BN, MA Nursing, PhD, FCNA(NZ) is Associate Professor in Nursing at Otago Polytechnic, Dunedin, New Zealand. She has more than 25 years' experience of working with the rural nursing workforce and community development research projects.

Samuel Mann (10000-0002-III8-7363) BSc(Hons), MSc, PhD, DipCertL\&T is a Professor in Leadership for Change and Professional Practice at Otago Polytechnic Dunedin. He has long worked in the area of sustainable practice, particularly in supporting complex decision making in rural communities.

Keith Whiddon (1D0000-0001-7337-0449) CertEd, BEd(Hons) originally a teacher of geography, spent several years leading on the strategic development of ICT in education as a school adviser and inspector. After moving to Bishop's Castle in 2014, Keith now lives as a musician and community activist. He is a school governor and chairs both the Bishop's Castle Community Partnership and the Michaelmas Fair.

Correspondence to: Jean Ross, Otago Polytechnic, Dunedin, New Zealand. Email: jean.ross@op.ac.nz

\section{REFERENCES}

Bergvall-Kåreborn, B., Ihlström Eriksson, C., Ståhlbröst, A., \& Svensson, J. (n.d.). A milieu for innovation - Defining living labs. Retrieved from http://www.diva-portal.org/smash/get/diva2:I004774/FULLTEXT0I.pdf

Couper, I., Strasser, R., Rourke, J., \& Wynn-Jones, J. (20I5). Rural health activism over two decades: The WONCA working party on rural practice 1992-2012. Rural and Remote Health, 15(3), 3245.

Doolan-Noble, F., Ross, J., Johnson, R., Birks, M., Francis, K. \& Mills, J. (2019). Rural nursing in Aotearoa New Zealand and Australia: Embracing strategic foresight to sustain tomorrow's workforce. Scope, Contemporary Research Topics: Health \& Wellbeing, 4, 58-62.

Gilbert, J. (2016). Leading in collaboration complex education systems. Education Council New Zealand. https://educationcouncil. org nz/sites/default/files/Five\%20Think\%20pieces.pdf

Hogarth, K., \& Rapata-Hanning, M. (20I5). Maori health in Aotearoa New Zealand. In J. Craft and C. J. Gordon (Eds), Understanding pathophysiology (pp. 1179-1197). Sydney, Australia: Elsevier Australia.

International Association for Community Development (IACD), (20I8). Towards shared international standards for community development practice. Retrieved from http://www.iacdglobal.org/

New Zealand Qualification Authority (n.d). Micro-credential pilot. Retrieved from https://www.nzqa.govt.nz/about-us/futurestate/quality-assurance/micro-credential-pilots/

Parsons DESIS Lab (n.d.). Parsons DESIS Lab. Retrieved from https://www.newschool.edu/desis/

Restieaux P., Norgate, A., Lyttle, C., Anderson, C., Monaghan, J., Baxter, J., Lucy ... Ross, J. (2020 in press). Sustainable Rural Futures A health perspective on Bishop's Castle, Shropshire, UK. Scope, Contemporary Research Topics, Health \& Wellbeing, 5, $194-205$.

Ross, J., \& Crawley, J. (Eds.). (2018). Stories of nursing in rural Aotearoa: A landscape of care. Dunedin, New Zealand: Rural Health Opportunities.

Ross, J., Crawley, J., \& Mahoney, L. (2017). Sustainable community development: Student nurses making a difference. Scope: Contemporary Research Topics: Learning \& Teaching, 4, 8-17.

Sustainable Development Goals (n.d.). Sustainable Development Goals. Retrieved from https://www. 17sustainabledevelopment goals.org/ 